Proc. 13th Econophysics Colloquium (EC) and 9th Symposium of Physics in Economy and Social Sciences (FENS), 2017

\title{
A Case Study of Diffusion of Innovation under Competition
}

\begin{abstract}
S. GÜNDÜÇ*
Ankara University Faculty of Engineering, Department of Computer Engineering, Gölbaşı, Ankara, Turkey

In this work, the aim is to discuss the relative importance of the driving parameters of the diffusion of innovation under competition. As a case study the competitive existence of Android and iOS operating systems are modeled in the context of an extended Bass model. The chosen model consists of two coupled differential equations. Each differential equation has the same form of the well known Bass equation with an extra term which represents the effect of the existence of the competitor. Since the smartphone sales and usage are globally well documented, publicly available global smartphone market share and sales data are used to determine the model parameter values. The smartphone global market share data spans 28 quarters starting from the first quarter of 2009. The distribution of the market share in the first quarter has been used as the initial values of the competing operating systems. A minimization process obtains the free parameters (six in total) of the differential equations. The values of the parameters are fixed by minimizing the difference between the solution of Bass equation and the smartphone global market share data. The parameter values of $\mathrm{iOS}$ and Android operating systems indicate that, on the worldwide scale, the adaptation of new ideas/products the dominantly led by the personal interactions. In fact, through social networks, information flow is immense, and individuals rely on the information obtained directly from a trusted individual. Publicity parameter values indicate that very rarely decision of an individual is formed by only the mass media or publicity. On the contrary, to the publicity parameter, it is observed that the existence of a competitor has a considerable effect on the sales of both parties.
\end{abstract}

DOI: 10.12693/APhysPolA.133.1465

PACS/topics: PACS/topics:02.30.Hq, 02.60.Cb,89.65.Gh

\section{Introduction}

The diffusion of innovation is of interest for both scientists and practising economists. It is a very challenging problem and has commercial value [1-3]. The diffusion of innovation studies aims to determine the conditions and dynamics of the spread of new ideas/products among the members of a society. Diffusion of innovation research can be conducted in two different approaches, namely macroscopic and microscopic modeling. When only the macroscopic effects are considered, the number of parameters which govern the dynamics are very limited. Microscopic models, on the other hand, consider individualsindividual and individual-environment interactions $[4,5]$.

The first mathematical model of the diffusion of innovation was introduced by Bass in 1969 [6]. Its success in explaining the spread of technological household goods opened a new era. Since then the advances in mathematical modeling of the diffusion of innovation made the subject more interesting and relatively more accurate predictions possible. Nevertheless the Bass model is still very successful in explaining dynamics of very different diffusion of innovation processes. For competitive markets, the well-known Bass model is extended to accommodate competition [7-9]. Also, the LotkaVolterra model [10, 11], which was initially used for the competing biological species, is reinterpreted in the terminology of competitive marketing. Recently both ex-

*e-mail: gunduc@ankara.edu.tr tended Bass model $[9,12-14]$ and the Lotka-Volterra model $[12,15,16]$ are used in the studies of diffusion of innovation process.

Since 2008, smartphone operating systems have shown a rapid change. This change has been subject to various studies $[12,16]$. Tseng et al. have studied competition among the existing operating systems by using an integrated model [12]. Tseng et al. have used scenario analysis, expert opinion, Delphi and extended diffusion of innovation techniques in their studies. Wang et al., on the other hand, used available sales data and the LotkaVolterra equation for short-term predictions for market shares of iOS and Android operating systems [16]. In the same work, equilibrium conditions of the Lotka-Volterra equation system have been studied.

In this work, the aim is to use the real data and the extended models of diffusion of innovation to understand the role of different effects on the market shares of various smartphone operating systems. The market share data of the smartphone operating systems start from the first quarter of 2009 and end at the beginning of 2016 . In between these dates quarterly data of both global unit sales and global market shares are published by Gartner [17] and also available from Statista $[18,19]$. At the beginning of 2009, there were more than 9 different operating systems. After 28 quarters there exists only two primary operating systems which are iOS and Android. Android operating system (first introduced in 2008) held less than $2 \%$ of the market shares at the beginning of 2009. This new operating system has gained ground in smartphone markets. In its expansion, all but one operating system, namely iOS lost their market shares. By the beginning of 2016, only Android and iOS operating systems shared the markets as approximately $90 \%$ and $10 \%$, respectively. 
The smartphone operating system market share data is an excellent playground for testing different models of diffusion of innovation and also discussing the conditions of competition and coexistence of different brands. The parameters of the model, the relative sizes of internal, external and the cross-brand terms are obtained by using publicly available market share data. The present work is divided into two sections: in the first part competitive existence of $\mathrm{iOS}$ and Android operating systems are discussed. In this discussion, both the Bass and LotkaVolterra equation are employed. In the second part, the success of iOS and Android operating systems resulted in the loss of market share for the other brands. One group of products gaining the control of all the markets is an example of distractive competition, which is similar extinction processes in biological systems. Some species diminish while the others dominate the environment. The dynamics of competition between old and new operating systems are discussed by using the Lotka-Volterra equation.

In the following section, the basics of the model will be introduced. The third section is devoted to the application of the model to real data on smartphones and the last section discusses the results and give hints for the further research.

\section{The model}

During the observation time of 28 quarters, the sales of smartphones have increased ten times. This growth rate in the sales is a clear indication of the growing potential market. Neither the Bass nor Lotka-Volterra models do consider a time dependence of the market growth. This problem can be partially overcome by using market shares data. The market share, $n_{i}(t)$ of product $i$ can be given as the ratio of the unit sales, $N_{i}(t)$, at time $t$ and the potential market size at the same time slice, $M(t)$, $n_{i}(t)=N_{i}(t) / M(t)$.

The extended Bass equation [7-9] in terms of market shares of two competing brands can be given as

$$
\begin{aligned}
& \frac{\mathrm{d} n_{1}(t)}{\mathrm{d} t}=\left[p_{1}+q_{1} n_{1}(t)+\gamma_{1,2} n_{2}(t)\right][1-n(t)], \\
& \frac{\mathrm{d} n_{2}(t)}{\mathrm{d} t}=\left[p_{2}+q_{2} n_{2}(t)+\gamma_{2,1} n_{1}(t)\right][1-n(t)] .
\end{aligned}
$$

Here $n_{i}(t)$ is the percentage of the adopters (market share) who adopted the brand $i, n(t)$ is the sum of market shares of both brands, $n(t)=n_{1}(t)+n_{2}(t)$. $p_{1}, q_{1}$ and $p_{2}, q_{2}$ are the innovation and imitation parameters of the first and the second brands respectively, $\gamma_{1,2}$ and $\gamma_{2,1}$ are the cross brand parameters.

In the same way the Lotka-Volterra $[10,11]$ equation is written in terms of competitive market shares

$$
\begin{aligned}
& \frac{\mathrm{d} n_{1}(t)}{\mathrm{d} t}=\left(a_{1}+b_{1} n_{1}(t)+c_{1,2} n_{2}(t)\right) n_{1}(t), \\
& \frac{\mathrm{d} n_{2}(t)}{\mathrm{d} t}=\left(a_{2}+b_{2} n_{2}(t)+c_{2,1} n_{1}(t)\right) n_{2}(t) .
\end{aligned}
$$

Here the number of each species at time $t$ is represented by $n_{i}(t), i=1,2$. Six parameters of the Lotka-Volterra equation controls the growth of each species, $a_{i}$, scale of the environment, $b_{i}$, and competition between the species, $c_{i, j}, i \neq j$. Survival of a brand depends on the correct environment and also competition ability with the existing rivals.

In the following section, the parameters, $p_{i}, q_{i}$ and $\gamma_{i, j}$, $i \neq j$, for the Bass equation and $a_{i}, b_{i}$ and $c_{i, j}, i, j=1,2$ coefficients for the Lotka-Volterra will be obtained by comparing the solutions of Eqs. (1) and (2) with the data.

\section{Results}

The market shares of different smartphone operating systems starting from the first quarter of 2009 are studied by using both Bass and Lotka-Volterra equations. The data obtained from Statista [18, 19] is used for both the initial values and the parameter fits. Global sales data and the global shares data (Fig. 1a and b) are available for 28 quarters between the years 2009 and 2016. In this study global share data is used for the parameter fits. The global market share data, exhibit the changes in the market share of the leading operating systems (Fig. 1b).
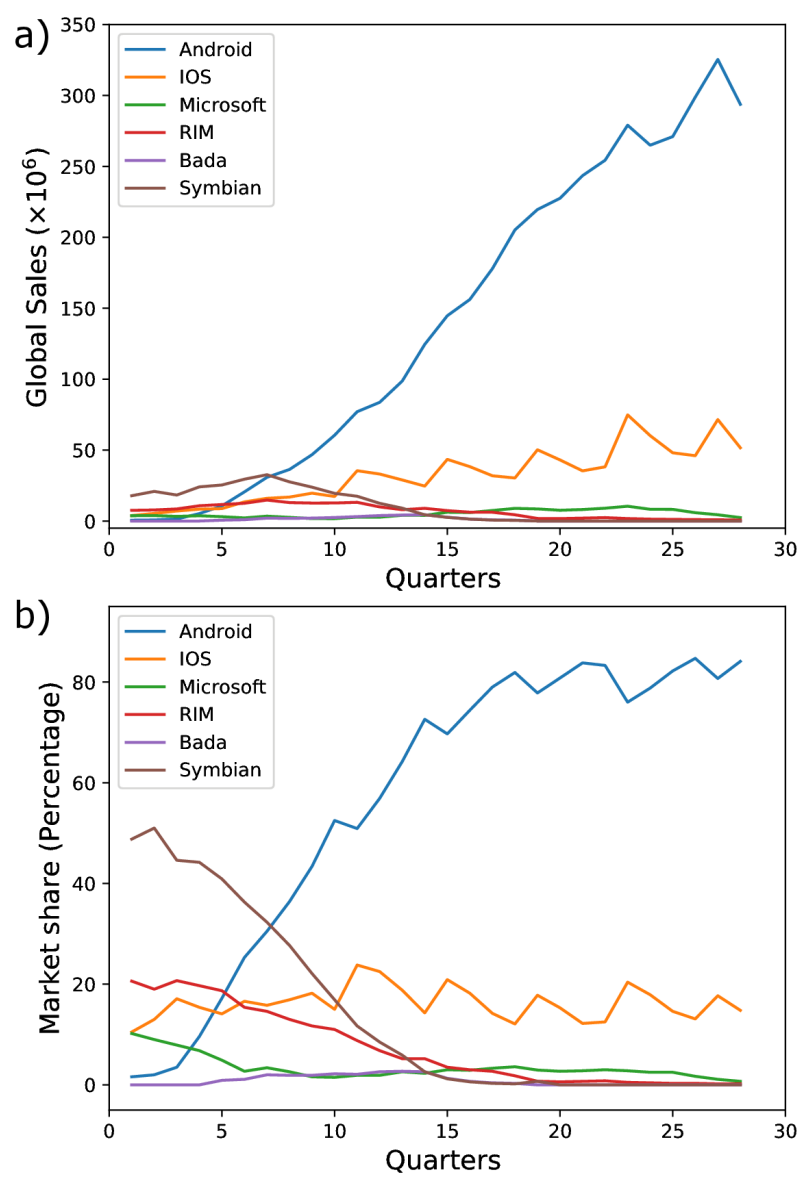

Fig. 1. Smartphone global market sales and shares data for a period of 28 quarters starting from the first quarter of 2009. (a) Global sales data, (b) global market share data. 
The total market share of Android and iOS operating systems both together was almost $12 \%$ at the beginning of 2009; 28 quarters later, at the beginning of 2016, markets were dominated by only two operating systems, namely Android and iOS.

To discuss effects of different elements on the diffusion of innovation the parameters of the Bass and LotkaVolterra equations are obtained by using a two-stage optimization program. For both equations, data fitting processes run into difficulties when the system approaches local minima. In search of global minima, randomly chosen points on parameter space are used as the initial values of the minimization routine. For minimizations, Python SciPy optimization packages are used. For each random initial parameter set, minimization routine (minimum, which is a part of Scipy optimization package) is used to obtain best parameter values which minimize the function by fitting the data with the theoretical model.

The fluctuations in sales data of both Android and iOS operating systems are closely related to the introduction of the new iOS models. In fact, the correlation between shares of Android and iOS operating system unit sales can easily be seen by comparing the data points in Fig. 1a and b. Since the sales of both operating systems are closely related, the sales/shares of both Android and iOS operating systems are considered together as a study of the diffusion of a new type of smartphone operating systems. The total smartphone market share expansion is represented by the Bass equation [6] with the parameters, $m_{(2016)}^{i O S+\text { Android }}=100 \%, p^{i O S+\text { Android }}=0.0106$ and $q^{i O S+A n d r o i d}=0.266$. As the smartphone markets are considered as a whole (Fig. 2a), imitation effects are the main driving force in the spread of the smartphones. When both operating systems are taken into consideration, mutual fluctuations smooths the shares data (Fig. 2a). Mutual cancellation of the fluctuations is an open indication of the cross-brand effects. Increase in the sales of one brand directly decreases the sales of the other brand. The competition between these two brands are also studied by using extended Bass Eq. (1) which accommodate two competitive brands in the same market.

The market share data is fitted by the six parameters of Eq. (1). Two sets of three parameters $p_{1}, q_{1}, \lambda_{1,2}$ and $p_{2}, q_{2}, \lambda_{2,1}$ are obtained by integrating the coupled differential equations and searching the parameter sets which minimize the distance between solutions, $n_{1}(t)$ and $n_{2}(t)$, and the data points. Figure $2 \mathrm{~b}$ shows the data points and the solution of the coupled Bass equation.

In the interacting system, both Android and iOS operating systems have very small innovation parameter while the imitation parameters seem to be the dominating factor (Table I). As far as the cross-brand effects are considered, the result indicates that the existence of iOS helps Android without being affected by the market share of Android operating system.

The Lotka-Volterra equation is well known as praypredator equation. Hence the Lotka-Volterra equation
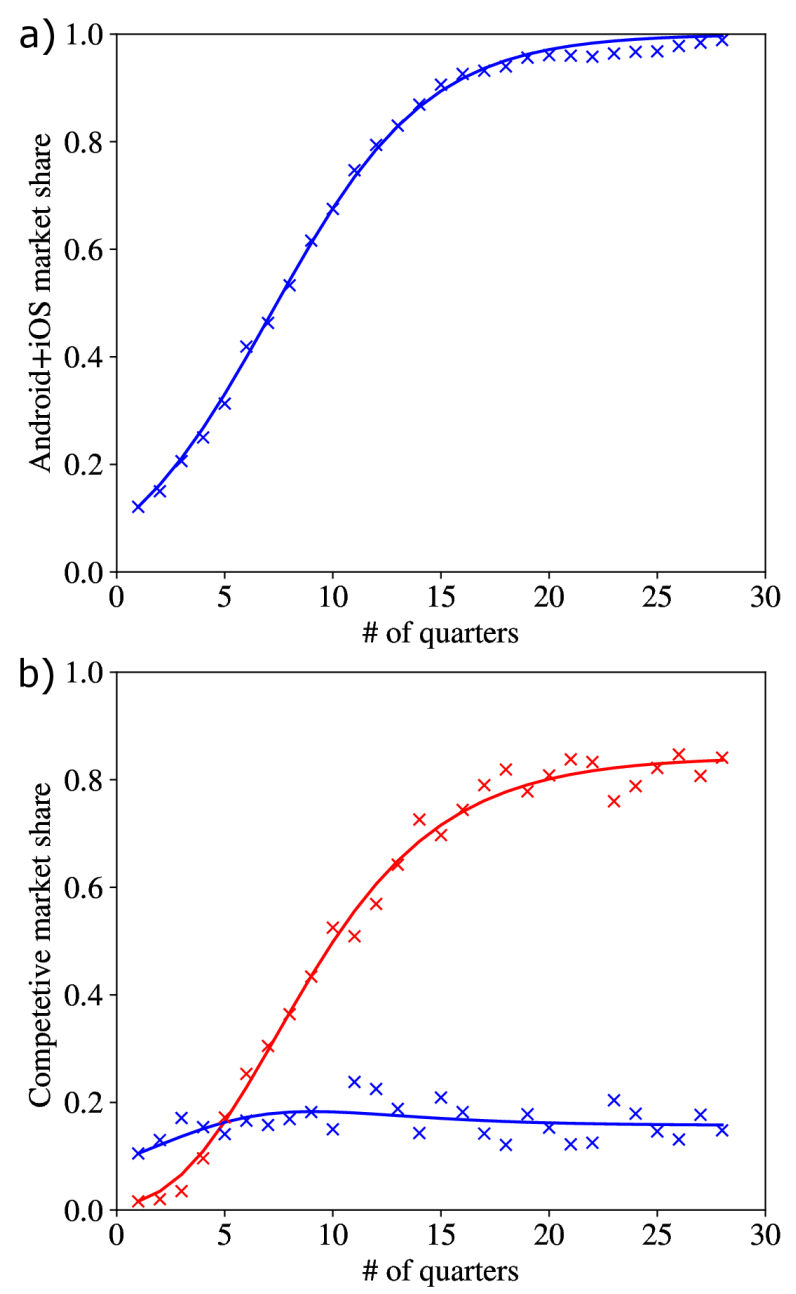

Fig. 2. Bass equation shares solutions for a period of 28 quarters starting from the first quarter of 2009. (a) Android and iOs total market share, (b) Android $+\mathrm{iOS}$ competitive coexistence.

TABLE I

Interacting market share results. Solution of extended Bass equation.

\begin{tabular}{c|c|c|c}
\hline \hline & $p$ & $q$ & $c$ \\
\hline Android & $-0.0519 \pm 0.0065$ & $0.2677 \pm 0.0049$ & $0.5941 \pm 0.0169$ \\
iOS & $0.0046 \pm 0.0077$ & $0.1380 \pm 0.0212$ & $-0.0682 \pm 0.0064$
\end{tabular}

is best suited for the competition between old operating systems which were existing at the initial stages (before 2009) and new, succeeding in ten years period. At the beginning of 2009, more than ten operating systems had their market share (Fig. 3). The market shares exhibited a rapid change. This change in the market resulted in that some operating systems have lost their share entirely while two, namely iOS and Android gained the markets. The Lotka-Volterra equation parameters (Table II) indicate that old operating systems have very high market growth parameter, at the same time market scale parameter is very high which explains the diminishing of the old 

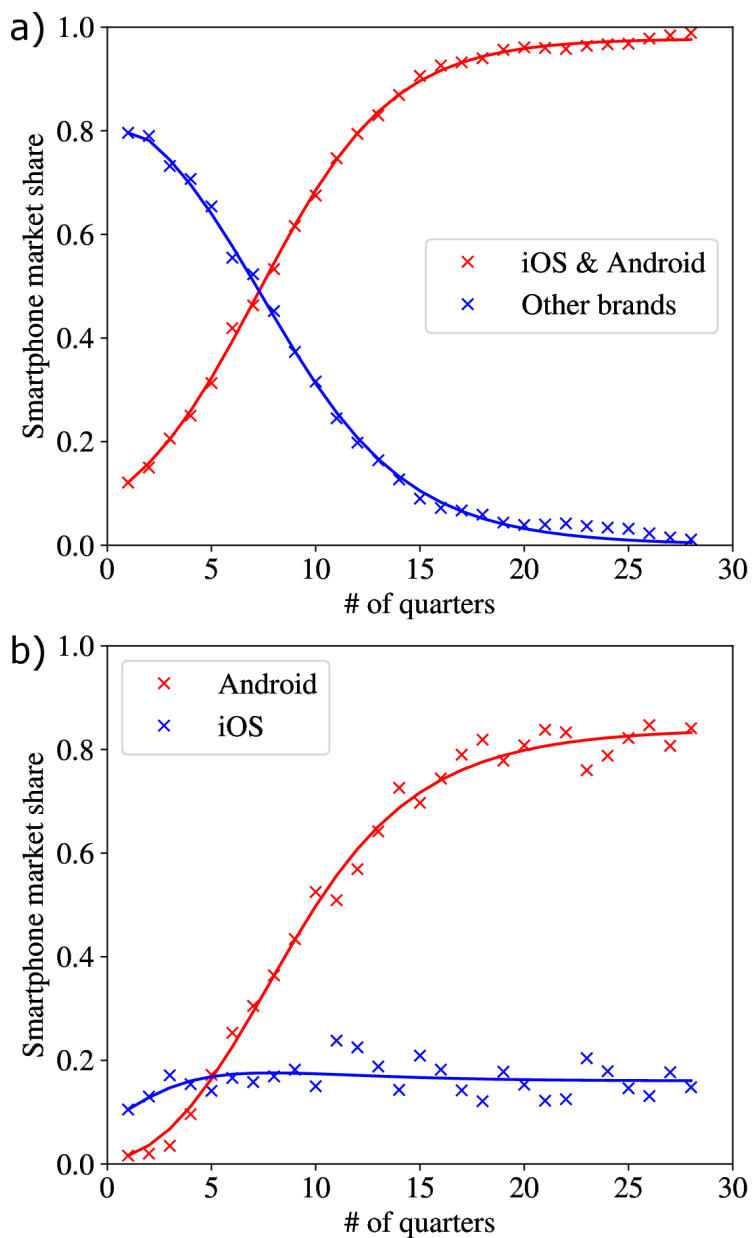

Fig. 3. Lotka-Volterra equation solutions of changing market shares for a period of 28 quarters starting from the beginning of 2009. (a) Android and iOS versus old operating systems, (b) the competitive coexistence of Android and iOS.

operating systems. Large negative effect of iOS and Android on the old operating systems is a characteristic of pray-predator relation.

TABLE II

iOS + Android vs. others: old operating systems lose their market share while iOS and Android gain and dominate the markets.

\begin{tabular}{c|c|c|c}
\hline \hline & $a$ & $b$ & $c$ \\
\hline Android \& iOS & $0.3334 \pm 0.0045$ & $0.3408 \pm 0.0045$ & $-0.01891 \pm 0.0043$ \\
Other brands & $6.5533 \pm 0.0660$ & $7.1040 \pm 0.0688$ & $-8.3970 \pm 0.07410$
\end{tabular}

The competition between iOS and Android operating systems also can be studied by using the Lotka-Volterra equation. Interpretation of the parameters of (2) is entirely different and complementary with the Bass equation. Significant market growth parameter is an indication of the gain of the Android operating system (Table III).
TABLE III

Lotka-Volterra equation solutions of coexistence of iOS and Android.

\begin{tabular}{c|c|c|c}
\hline \hline & $a$ & $b$ & $c$ \\
\hline Android & $2.5302 \pm 0.0262$ & $1.2274 \pm 0.0148$ & $-9.3586 \pm 0.0556$ \\
iOS & $0.5481 \pm 0.0056$ & $2.9598 \pm 0.0154$ & $-0.0880 \pm 0.0037$
\end{tabular}

The results are discussed in the following conclusion section.

\section{Conclusions}

The mobile phones created entirely different channels of communication and enriched the means of social interactions. A boom in social media has been experienced by the introduction of the smartphone. A decade ago inevitable switch from the mobile phones to smartphones was an exceptional example of the diffusion of innovation. First of all, some of the mobile phone producers could not coop with the new trend, and they lost their share in the market. Secondly, some surviving brands cooped with the latest developments and shared the market with competitors whose coexistence helped all parties. One of the main conclusions of this work is that the existence of the competitors helped all parties, word-of-mouth became the most crucial factor for both to boost the sales and the growth of markets.

The market share growth dynamics of iOS and Android systems can be best studied by using Bass, extended Bass, and Lotka-Volterra equations. In such studies, the major concern must be the relevance of the solutions with the related market parameters. Both the Bass and Lotka-Volterra equations have two sets of parameters. The first set is related to the brand itself: growth and market size parameters for the Lotka-Volterra and innovation and imitation parameters for Bass equations. The second set of parameters are related to the inter-brand interactions for both equations. The values of both interbrand and cross-brand parameters have correct signs and sizes (Tables I, III, II). The observed market behavior (Fig. 3) is well explained in terms of the obtained parameters. The Lotka-Volterra equation best represents the period between 2009 and 2014 when the old operating system brands lose their market shares in favor of iOS and Android operating systems. In this time span, a pray-preditor situation can explain the type of competition in which the major operating systems leave their market shares to two relatively new operating systems. From the obtained inter-brand parameters, the market share gain of iOS and Android can be interpreted as pray-predator relation (2). On the other hand, the competition between iOS and Android operating systems are better suited to modeling by extended Bass type equations. The existence of one helps the other to expand its market share. 


\section{References}

[1] V. Mahajan, E. Muller, Y. Wind, New-Product Diffusion Models, Kluwer Academic Press, 2000.

[2] E. Rogers, Diffusion of Innovations, 5th ed., Free Press, New York 2003.

[3] N. Meade, T. Islam, Int. J. Forecast. 22, 519 (2006).

[4] W. Rand, R.T. Rust, Int. J. Res. Market. 28, 181 (2011).

[5] A. Kowalska-Pyzalska, K. Ćwik, A. Jedrzejewski, K. Sznajd-Weron, Acta Phys. Pol. A 129, 1055 (2016).

[6] F.M. Bass, Manag. Sci. 15, 215 (1969).

[7] R.A. Peterson, V. Mahajan, in: Multi-Product Growth Models. Reseach in Marketing, Ed. J. Shethe, JAI Press, Greenwich CT 1978.

[8] V. Mahajan, E. Muller, F. Bass, J. Market. 54, 1 (1990).

[9] B. Libai, E. Muller, R. Peres, J. Market. 73, 19 (2009).

[10] A.J. Lotka, Elements of Physical Biology, William \& Wilkins Co., Baltimore (MD) 1925.
[11] V. Volterra, Variations and fluctuations of the number of individuals in animal species living together, In Chapman, R. N. Animal Ecology. McGraw-Hill 1931.

[12] F.-M. Tseng, Y.-L. Liu, H.-H. Wu, J. Eng. Technol. Manage. 32, 40 (2013).

[13] C.E. Laciana, G. Gual, D. Kalmus, N. Oteiza-Aguirre, S.L. Rovere, Physica A 413, 104 (2014).

[14] R. Guseo, C. Mortarino, Ann. Appl. Statist. 9, 2073 (2015).

[15] Jonghwa Kim, Deok-Joo Lee, Jaekyoung Ahn, Comput. Industr. Eng. 51, 174 (2006).

[16] Hsi-Tse Wang, Ta-Chung Wang, Technol. Anal. Strateg. Manage. 29, 414 (2016).

[17] Gartner Press Release Archive.

[18] Global market share held by the leading smartphone operating systems in sales to end users from 1st quarter 2009 to 1st quarter 2018, Statista, The Statistics Portal.

[19] Global smartphone sales to end users from 1st quarter 2009 to 1st quarter 2018, by operating system (in million units), Statista, The Statistics Portal. 